\title{
Perfil epidemiológico do trauma ocular penetrante antes e após o novo código de trânsito
}

\author{
Epidemiological profile of penetrating ocular trauma before and after the new \\ trafficcode
}

\author{
Paulo Caldas Silber ${ }^{1}$ \\ Luciene Barbosa de Souza ${ }^{2}$ \\ Maira Tiyomi Sacata Tong $\mathbf{u}^{3}$
}

${ }^{1}$ Estagiário do Pronto Socorro da Universidade Federal de São Paulo (UNIFESP).

${ }^{2}$ Doutora em Oftalmologia pela Universidade Federal de São Paulo (UNIFESP).

${ }^{3}$ Pós-graduanda nível doutorado pela Universidade Federal de São Paulo (UNIFESP).

Endereço para correspondência: Av. Coronel Marcos, 419 - Porto Alegre (RS) CEP 91760-000.

E-mail: pcsilber@terra.com.br

Recebido para publicação em 20.12.2000 Aceito para publicação em 20.12.2001

\section{RESUMO}

Objetivo: Comparar o perfil epidemiológico dos pacientes com trauma ocular penetrante (TOP) antes e após a regulamentação do novo código de trânsito. Métodos: Estudo retrospectivo de 253 pacientes com TOP examinados na Seção de Trauma Ocular (UNIFESP) de janeiro de 1997 a abril de 1999. Os pacientes foram divididos em dois grupos: Grupo I, pacientes com trauma ocular antes da implantação do novo código; Grupo II, história de trauma após sua implantação. Os pacientes foram avaliados em relação a diferentes aspectos do trauma e exame oftalmológico. Resultados: Os achados epidemiológicos em relação à idade, sexo e raça foram similares em ambos os grupos. No grupo I, os pacientes entre $21 \mathrm{e}$ 50 anos apresentaram distribuição similar quanto à etiologia do trauma, ao passo que no grupo II, no mesmo intervalo de idade, predominaram os acidentes automobilísticos. Em relação ao uso do cinto de segurança, $60 \%$ e $92 \%$ dos pacientes não estavam usando o cinto, nos grupos I e II, respectivamente. $60 \%$ dos pacientes no grupo II mencionaram consumo de álcool, contra 40\%, no grupo I. Conclusão: Apesar das medidas de impacto tomadas pelo governo para controlar os acidentes, os danos do trauma ocular continuam relacionados a fatores passíveis de prevenção, como o uso do cinto de segurança e consumo de álcool.

Descritores: Traumatismos oculares/epidemiologia; Acidentes de trânsito/prevenção \& controle; Cintos de segurança; Estudos retrospectivos

\section{INTRODUÇÃO}

$\mathrm{O}$ trauma ocular representa freqüente causa de atendimento nos prontosocorros de oftalmologia do mundo inteiro. Estudos americanos mostram que é a causa mais comum de cegueira unilateral, sendo responsável por $7 \%$ da cegueira bilateral na faixa etária de 20 a $64 \operatorname{anos}^{(1-2)}$.

Em 1980, a Sociedade Americana de Prevenção da Cegueira estimou que $55 \%$ dos casos de trauma ocular ocorrem em indivíduos com idade abaixo de 25 anos e que um terço dos indivíduos que perdem a visão por trauma ocular estão na primeira década de vida ${ }^{(3-4)}$.

A causa das perfurações oculares tem variado conforme a faixa etária, mostrando uma distribuição bimodal. Entre as crianças, os acidentes domésticos e atividades de lazer compreendem a maioria dos $\operatorname{casos}^{(5)}$. Acidentes automobilísticos, traumatismos ocupacionais e violência constituem as principais causas de lesões oculares em adultos jovens ${ }^{(5-6)}$.

A epidemiologia do trauma de face é dinâmica sofrendo interferência de medidas de impacto, como a obrigatoriedade no uso do cinto de segurança ${ }^{(7)}$. 
Em São Paulo, foi instituída a obrigatoriedade do uso do cinto de segurança através de uma lei municipal, em 1994. Soriano e cols. relataram diminuição de $4,9 \%$ na freqüência de perfurações oculares por acidentes automobilísticos após a implantação dessa lei ${ }^{(8)}$.

Em 1997, foi aprovado o novo Código Nacional de Trânsito (CNT) e a partir de abril de 1998 a punição pela falta do uso do cinto de segurança entrou em vigor.

Com o objetivo de estudar e comparar o perfil epidemiológico do traumatismo ocular penetrante, antes e após a vigência do novo Código Nacional de Trânsito na cidade de São Paulo, foi realizado este estudo retrospectivo de pacientes atendidos em um serviço de referência terciária.

\section{MÉTODOS}

Foi realizado um estudo retrospectivo dos prontuários de 253 pacientes, vítimas de traumatismo ocular penetrante, atendidos no Setor de Trauma Ocular do Departamento de Oftalmologia da Universidade Federal de São Paulo (UNIFESP).

Os atendimentos ocorreram no período de janeiro de 1997 a abril de 1999, sendo excluído o intervalo entre janeiro de 1998 a abril de 1998, por corresponder ao período de adaptação ao novo código de trânsito. Os pacientes foram divididos em duas amostras. A amostra I, constituída pelos pacientes atendidos de janeiro de 1997 a janeiro de 1998, quando a obrigatoriedade do uso do cinto de segurança já existia, porém não havia as penalidades impostas por um código de trânsito. Amostra II, constituída por pacientes atendidos de abril de 1998 a abril de 1999, quando foi implementado o novo código nacional de trânsito. As duas amostras foram comparadas quanto ao sexo, idade, raça, olho afetado, causa do traumatismo (automobilístico, violência, doméstico, esporte/lazer e trabalho). Nos casos de acidentes automobilísticos, foram avaliados o horário do acidente, tipo do veículo, uso de cinto de segurança, uso de bebidas alcoólicas, localização do paciente dentro do veículo e olho acometido.

\section{RESULTADOS}

Foram analisados 253 prontuários, 140 na amostra I e 113 na amostra II. A distribuição por sexo está demonstrada na tabela 1, onde podemos observar predominância do sexo masculino nas duas amostras, na proporção de 4:1.

\begin{tabular}{|c|c|c|c|}
\hline Sexo & Amostra I & Amostra II & Total \\
\hline Masculino & $116(82,9 \%)$ & $90 \quad(79,6 \%)$ & $206(80,1 \%)$ \\
\hline Feminino & $24(17,1 \%)$ & $23(20,4 \%)$ & $47 \quad(20,9 \%)$ \\
\hline Total & $140(100 \%)$ & $113(100 \%)$ & $253(100 \%)$ \\
\hline
\end{tabular}

A média de idade da amostra I foi de 27,4 anos e desvio padrão de 17,2 anos, com idade variando de 2 a 81 anos; na amostra II, a média de idade foi 26 anos e desvio padrão de 15,9 anos, variando de 1 a 76 anos.

Em relação ao olho afetado, houve distribuição uniforme nas duas amostras, direito e esquerdo, com similar acometimento bilateral, 4 na amostra I e 5 na amostra II. Em relação à raça não houve diferença entre a negra e branca. Foi encontrado somente um paciente da raça amarela na amostra II.

A distribuição em relação à faixa etária está demonstrada na tabela 2 .

A distribuição da faixa etária em relação à causa nas duas amostras está demonstrada na tabela 3 e 4 .

Em relação aos traumatismos associados a acidentes automobilísticos, observamos:

- O automóvel foi o meio de transporte mais freqüentemente associado ao trauma, representando $91 \%$ e $88 \%$, respectivamente na amostra I e II.

Quanto à posição da vítima dentro do veículo, todos encontravam-se nos acentos dianteiros, com número de vítimas

\begin{tabular}{|c|c|c|}
\hline Faixa etária & Amostra I & Amostra II \\
\hline $0-12$ & $33(23,6 \%)$ & $30 \quad(26,5 \%)$ \\
\hline $13-20$ & $16 \quad(11,4 \%)$ & $16 \quad(14,2 \%)$ \\
\hline $21-50$ & $76 \quad(54,3 \%)$ & $60 \quad(53,1 \%)$ \\
\hline+50 & $15(10,7 \%)$ & $07 \quad(6,2 \%)$ \\
\hline Total & $140(100 \%)$ & $113(100 \%)$ \\
\hline
\end{tabular}

\begin{tabular}{|c|c|c|c|c|c|}
\hline $\begin{array}{l}\text { Faixa } \\
\text { etária }\end{array}$ & Violência & Doméstico & $\begin{array}{l}\text { Automo- } \\
\text { bilístico }\end{array}$ & Trabalho & Total \\
\hline $0-12$ & 8 & 24 & 0 & 01 & 33 \\
\hline $13-20$ & 03 & 09 & 02 & 02 & 16 \\
\hline $21-50$ & 10 & 22 & 20 & 24 & 76 \\
\hline+50 & 02 & 05 & 02 & 06 & 15 \\
\hline Total & 23 & 60 & 24 & 33 & 140 \\
\hline
\end{tabular}

\begin{tabular}{|c|c|c|c|c|c|}
\hline $\begin{array}{l}\text { Faixa } \\
\text { etária }\end{array}$ & Violência & Doméstico & $\begin{array}{l}\text { Automo- } \\
\text { bilístico }\end{array}$ & Trabalho & Total \\
\hline $0-12$ & 04 & 23 & 01 & 01 & 30 \\
\hline $13-20$ & 02 & 08 & 02 & 04 & 16 \\
\hline $21-50$ & 05 & 12 & 27 & 15 & 59 \\
\hline+50 & 0 & 01 & 01 & 05 & 07 \\
\hline Total & 11 & 44 & 31 & 25 & 113 \\
\hline
\end{tabular}




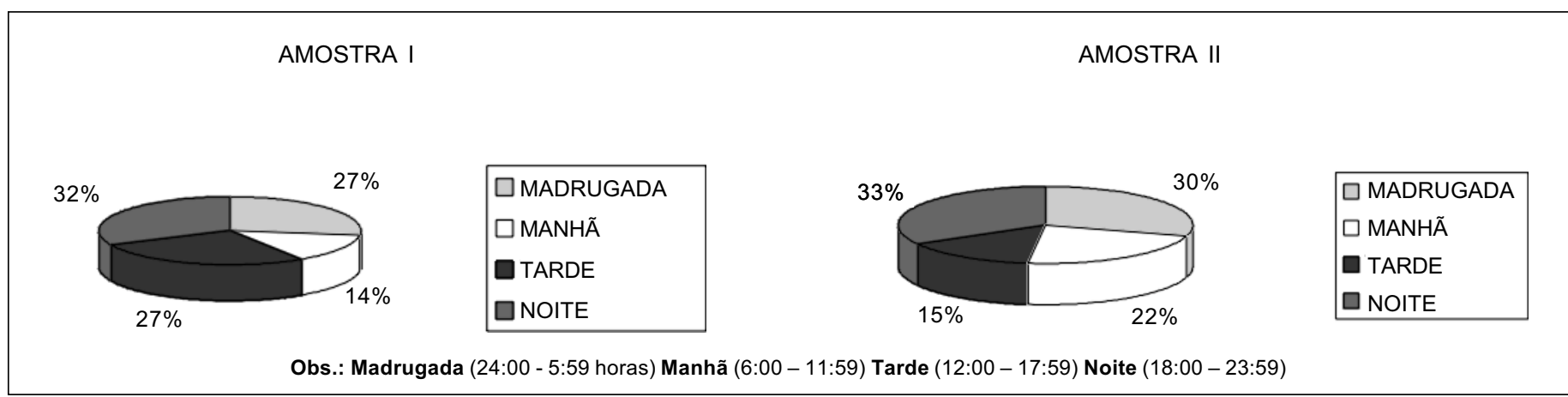

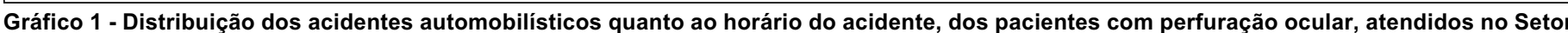
de Trauma Ocular do Departamento de Oftalmologia da UNIFESP. São Paulo - Brasil, abril de 1998 a abril 1999

equivalentes entre o acento do motorista e acompanhante da frente nas amostras I e II, respectivamente.

- Os acidentes ocorreram principalmente à noite e na madrugada nas duas amostras.(Gráfico 1)

- A distribuição do uso do cinto de segurança nas duas amostras está demonstrada na tabela 5 .

- A ingestão de álcool foi relatada em 37\% na amostra I e $62 \%$ na II, conforme podemos observar no gráfico 2 .

\section{DISCUSSÃO}

Este estudo mostrou predominância do sexo masculino, na proporção de 4:1 nas duas amostras, dados semelhantes aos encontrados na literatura ${ }^{(6,9,13)}$. Esta predominância se deve provavelmente ao fato dos homens serem mais expostos aos acidentes automobilísticos, trabalho, violência e esporte.

Tabela 5. Distribuição do uso do cinto nas duas amostras, dos pacientes atendidos no Setor de Trauma Ocular do Departamento de Oftalmologia da UNIFESP. São Paulo - Brasil, abril de 1998 a abril 1999

\begin{tabular}{llllll} 
Cinto & \multicolumn{2}{c}{ Amostra I } & \multicolumn{2}{c}{ Amostra II } & \multicolumn{2}{c}{ Total } \\
Não & $12(60 \%)$ & $23(92 \%)$ & 35 & $(77,7 \%)$ \\
Sim & $08(40 \%)$ & $02(8 \%)$ & 10 & $(23,3 \%)$ \\
Total & $20(100 \%)$ & $25(100 \%)$ & $45(100 \%)$ \\
\hline
\end{tabular}

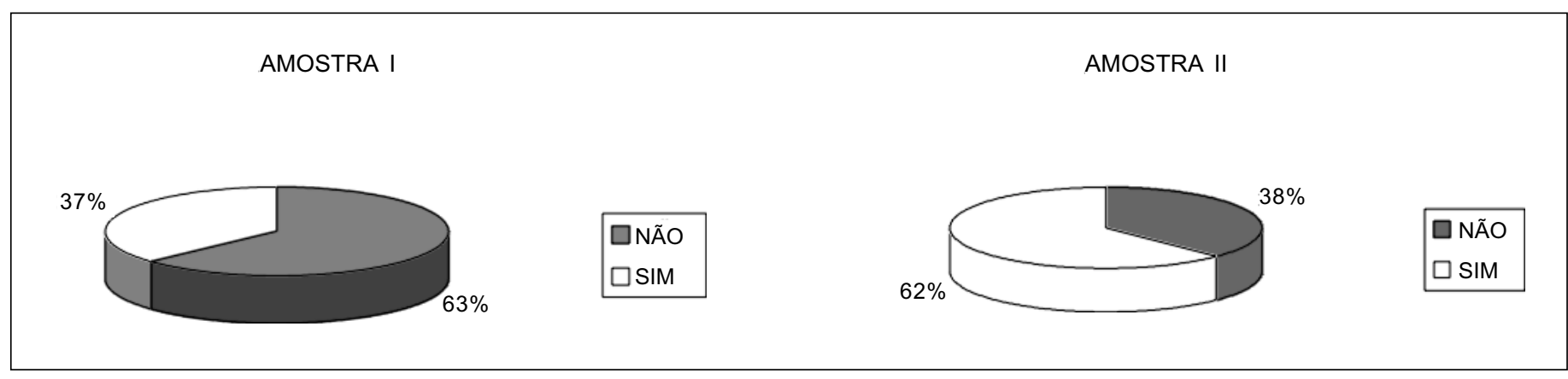

Gráfico 2 - Distribuição dos acidentes automobilísticos quanto à ingestão de álcool, dos pacientes com perfuração ocular, atendidos no Setor

de Trauma Ocular do Departamento de Oftalmologia da UNIFESP. São Paulo-Brasil, abril de 1998 a abril 1999
A faixa etária mais acometida foi o adulto, também de acordo com os dados da literatura ${ }^{(6,9)}$.

Em relação à causa, os acidentes automobilísticos foram a terceira causa de perfuração ocular na amostra I e a segunda na amostra II. Observamos, em nossa amostra que, apesar da regulamentação da obrigatoriedade do uso do cinto de segurança, com aumento das punições com o novo código de trânsito houve maior número de casos de perfuração ocular por acidente automobilístico. Estudos realizados, neste serviço, por Soriano e col. relataram queda de $34 \%$ nas causas de perfuração por acidente automobilístico após a lei municipal de $1994^{(8)}$. No entanto, Carani e col. descreveram os acidentes automobilísticos como principal causa de perfuração ocular no período entre maio de 1996 e março de $1997^{(10)}$. Nosso resultado pode ser explicado por tratar-se de um serviço de referência que drena casos mais graves, ou nossa amostra não represente a população geral de forma significativa.

Levando em consideração somente os acidentes automobilísticos foram analisados horário do acidente, tipo de veículo, posição do paciente no veículo, uso de cinto de segurança e ingestão de álcool. A maioria dos acidentes ocorreram no período da noite e madrugada ( 32 e $27 \%$ na amostra I e 33 e $30 \%$ na amostra II), semelhante ao encontrado por Viana e col. ${ }^{(6)}$. No período noturno (18h-6h) os acidentes ocorrem mais, possivelmente, porque a imprudência é mais freqüente 
pelo abuso de bebidas alcoólicas, pelo policiamento menos ostensivo, ou pelo próprio cansaço físico.

A maioria dos acidentes ocorreu em automóveis nas duas amostras. Somente um acidente em trator e um em caminhão e dois em motocicleta nos dois grupos. Quanto à posição do paciente no veículo no momento do acidente, aproximadamente $50 \%$ dos pacientes estavam no banco do motorista e $50 \%$ no banco do passageiro, nas duas amostras. Estudo realizado em 1988 mostrou que a maioria dos acidentados encontravam-se na posição de passageiro ${ }^{(6)}$. Gemperli et al. em estudo mais recente, mostrou que com o uso de cinto de segurança a posição do paciente não apresentava diferença estatística no banco dianteiro. Sem a utilização do cinto, havia predominância dos acidentados na posição de acompanhante ${ }^{(7)}$. Kara José e cols. encontraram maior vulnerabilidade dos acompanhantes do banco dianteiro seguidos da posição de motorista ${ }^{(9)}$.

Foi observado, quanto ao uso de cinto de segurança, que, nesta amostra, com os grupos divididos em um período antes e um período após a implantação das punições, encontramos um maior número de pacientes que não usavam cinto (de 60 para $92 \%)$. O mesmo ocorreu com a ingestão de álcool passando de $37 \%$ para $62 \%$. Vianna e cols. descreveram que $28,3 \%$ dos pacientes haviam ingerido algum tipo de bebida alcoólica ${ }^{(6)}$.

\section{CONCLUSÃO}

Esses dados não querem dizer ou insinuar que a implementação do novo código acarretou alteração no comportamento das pessoas a ponto de observarmos diminuição do uso do cinto e aumento da ingestão de álcool. Devemos lembrar que o número de pacientes estudados não representa de maneira significativa a população geral. Além disso, o estudo foi realizado em um hospital de referência terciária em oftalmologia na cidade, o que dificulta extrapolar os resultados para toda a população da cidade. Mesmo assim, com esse estudo lembramos a importância de campanhas de educação e conscientização da população sobre esse grave problema.

\section{ABSTRACT}

Purpose: To study the epidemiologic profile of the patients with penetrating ocular trauma (POT) before and after the application of the new traffic code. Methods: Retrospective study of 253 patients with POT examined at the Ocular Trauma
Section (UNIFESP) from January 1997 to April 1999. The patients were divided into 2 groups: Group I, patients with ocular trauma before the new traffic code; Group II, trauma history after the new code. The patients were evaluated regarding different aspects on trauma and ophthalmic evaluation. Results: The epidemiological findings regarding age, sex and race were similar in both groups. In group I, the patients between 21-50 years presented similar trauma etiology distribution, while in group II, in the same age range, they presented predominance of automotive-related trauma. Regarding seat belt use, $60 \%$ and $92 \%$ of patients were not using seat belts at the time of the trauma in groups I and II, respectively. $60 \%$ of the patients in group II mentioned alcohol consumption against $40 \%$ in group I. Conclusion: Besides the impact measures taken by state to control accidents, ocular trauma damages continue to be related to preventable risk factors like seat belt use and alcohol consumption.

Keywords: Eye injuries / epidemiology; Traffic accidents / prevention \& control; Seat belts; Retrospective studies

\section{REFERÊNCIAS}

1. Kuhn F, Morris R, Witherspoon D, Heimann K, Jeffers JB, Treister G. A standardized classification of ocular trauma. [commented on Ophthalmology 1997;104:344-5]. Ophthalmology 1996;103:240-3.

2. Pieramici DJ, Sternber Jr. P, Aaberg TM, Bridges WZ, Capone A, Cardillo JA, et al. Perspective - A system for classifying mechanical injuries of the eye (Globe). The ocular trauma classification Group [commented on Am J Ophthalmol 1998;125:565-6]. Am J Ophthalmol 1997;123:820-31.

3. Erwin-Mulvey LD, Nelson LB, Freeley DA. Pediatric eye trauma. Pediatr Clin North Am 1983;30:1167-83.

4. National Society to Prevent Blindness. Vision problems in the United States. New York; 1980. p.32-3.

5. Alves RM, Kara-Jose N. O trauma ocular como causa de cegueira. Rev Méd (São Paulo);1997;76:297-302.

6. Vianna Filho RG, Souza LB, Bordon AF, Freitas D. Estudo epidemiológico das perfurações oculares em acidentes automobilísticos. Arq Bras Oftalmol 1995;58:460-4.

7. Gemperly R, Lourenço LA, Lee YS, Ferreira MC, Lodovici O. A utilização do cinto de segurança na prevenção das fraturas de face. Rev Hosp Clín Fac Med Univ São Paulo 1990;45:263-7.

8. Soriano ES, Sousa LB, Moraes NSB, Freitas D. Incidência de perfuração ocular em acidentes automobilístico pré e pós-implantação da lei de obrigatoriedade do uso de cinto de segurança na cidade de São Paulo. Arq Bras Oftalmol 1996;59:382.

9. Kara-José N, Alves MR, Sampaio MW, Bonanomi MTBC. Ferimentos perfurantes do globo ocular por acidentes automobilísticos. Bol Oficina Sanit Pananam 1983;95:547-55.

10. Carani JCE, Machado CG, Gomi CF, Carvalho RMS. Ferimentos perfurantes oculares no Hospital das Clínicas da Faculdade de Medicina da Universidade de São Paulo. O que mudou nos últimos 27 anos. Arq Bras Oftalmol 1999;62:310-4. 